\title{
Genome-wide association reveals host-specific genomic traits in Escherichia coli
}

Sumeet K. Tiwari ${ }^{{ }^{*}}$,Boas C.L. van der Putten ${ }^{2,3^{*}}$, Thilo M. Fuchs ${ }^{4}$, Trung N. Vinh ${ }^{5}$, Martin Bootsma $^{6}$, Rik Oldenkamp ${ }^{2}$, Roberto La Ragione ${ }^{7}$, Sebastien Matamoros ${ }^{3}$, Ngo T. Hoa ${ }^{5,8,9}$, Christian Berens ${ }^{4}$, Joy Leng ${ }^{7}$, Julio Álvarez ${ }^{10,11}$, Marta Ferrandis-Vila ${ }^{4}$, Jenny M. Ritchie ${ }^{12}$, Angelika Fruth $^{13}$, Stefan Schwarz ${ }^{14}$, Lucas Domínguez ${ }^{10,11}$, María Ugarte-Ruiz ${ }^{10}$, Astrid Bethe $^{14}$, Charlotte Huber ${ }^{15}$, Vanessa Johanns ${ }^{15}$, Ivonne Stamm ${ }^{16}$, Lothar H. Wieler ${ }^{17}$, Christa Ewers $^{18}$, Amanda Fivian-Hughes ${ }^{12}$, Herbert Schmidt ${ }^{19}$, Christian Menge ${ }^{4}$, Torsten Semmler ${ }^{1 \#,}$ Constance Schultsz ${ }^{2,3 \#}$

${ }^{1}$ Robert Koch Institute, Genome sequencing and genomic epidemiology, Berlin, Germany

${ }^{2}$ Amsterdam UMC, Department of Global Health, Amsterdam, Netherlands

${ }^{3}$ Amsterdam UMC, Department of Medical Microbiology, Amsterdam, Netherlands

${ }^{4}$ Friedrich-Loeffler-Institut, Institute of Molecular Pathogenesis, Jena, Germany

${ }^{5}$ Oxford University Clinical Research Unit, Vietnam

${ }^{6}$ UMC Utrecht, Utrecht, Netherlands

${ }^{7}$ Department of Pathology and Infectious Diseases, School of Veterinary Medicine, University of Surrey, Guildford, UK

${ }^{8}$ Nuffield Department of Medicine, University of Oxford, Oxford OX3 7BN, UK

${ }^{9}$ Microbiology Department and the Micro-Parasitology Unit of the Center for Bio-Medical

Research, Pham Ngoc Thach University of Medicine, Ho Chi Minh City, Vietnam

${ }^{10}$ VISAVET Health Surveillance Centre, Complutense University of Madrid, Madrid, Spain

${ }^{11}$ Department of Animal Health, Faculty of Veterinary Medicine, Complutense University of

Madrid, Madrid, Spain

${ }^{12}$ School of Biosciences and Medicine, University of Surrey, Guildford, UK

${ }^{13}$ Robert Koch Institute, Enteropathogenic Bacteria and Legionella, Wernigerode, Germany

${ }^{14}$ Institute of Microbiology and Epizootics, Freie Universität Berlin, Berlin, Institute of

Microbiology and Epizootics, Berlin, Germany

${ }^{15}$ Robert Koch Institute, Advanced Light and Electron Microscopy, Berlin, Germany

${ }^{16}$ Vet Med Labor GmbH, Division of IDEXX Laboratories, Ludwigsburg, Germany

${ }^{17}$ Robert Koch Institute, Berlin, Germany

${ }^{18}$ Institute of Hygiene and Infectious Diseases of Animals, Giessen, Germany

${ }^{19}$ Institute of Food Science and Biotechnology, Department of Food Microbiology and

Hygiene, University of Hohenheim, Stuttgart, Germany

${ }^{*}$ These authors contributed equally to this work

${ }^{\#}$ Corresponding authors 


\section{Abstract:}

Escherichia coli is an opportunistic pathogen that can colonize or infect various host species.

There is a significant gap in our understanding to what extent genetic lineages of E. coli are adapted or restricted to specific hosts. In addition, genomic determinants underlying such host specificity are unknown.By analyzing a randomly sampled collection of 1198 whole-genome sequenced E. coli isolates from four countries (Germany, UK, Spain, and Vietnam), obtained from five host species (human, pig, cattle, chicken, and wild boar) over 16 years, from both healthy and diseased hosts, we demonstrate that certain lineages of E. coli are frequently detected in specific hosts. We report a novel nan gene cluster, designated nan-9, putatively encoding acetylesterases and determinants of uptake and metabolism of sialic acid, to be associated with the human host as identified through genome wide association studies. In silico characterization predicts nan-9 to be involved in sialic acid (Sia) metabolism. In vitro growth experiments with a representative $\Delta$ nan E. coli mutant strain, using sialic acids 5 - $N$-acetyl neuraminic acid (Neu5Ac) and $N$-glycolyl neuraminic acid (Neu5Gc) as the sole carbon source, indicate an impaired growth behaviour compared to the wild-type. In addition, we identified several additional $E$. coli genes that are potentially associated with adaptation to human, cattle and chicken hosts, but not for the pig host. Collectively, this study provides an extensive overview of genetic determinants which may mediate host specificity in E. coli. Our findings should inform risk analysis and epidemiological monitoring of (antimicrobial resistant) E. coli. 


\section{Introduction:}

Escherichia coli is a Gram-negative bacterium which has been isolated from various host species, including humans, cattle, chickens and pigs(1). Because E. coli can colonize or infect multiple host species, this bacterium can act as a reservoir for genes encoding antimicrobial resistance $(\mathrm{AMR})(2)$ that can be transmitted between different host species. The likelihood that E. coli and its AMR encoding genes persist in a new host after transmission depends on multiple factors(3,4). For example, small changes in metabolic pathways may enable E. coli to colonize or infect a host more efficiently(1). Several studies have suggested that highly successful E. coli clones, such as the sequence type 131 (ST131) clone(5,6) or clonal complex 87 (ST58 and ST155) E. coli facilitate the spread of AMR E. coli in the human population(7) whilst other studies have shown that different lineages of AMR E. coli vary in their ability to spread(8). These findings both indicate that AMR genes, at least to some extent, hitchhike on bacterial strains that are specifically equipped to colonize a given host. Beyond classical virulence or adhesion factors, genetic and functional traits defining different degrees of host adaptation $(3,9)$ and thereby indirectly impacting on the spread of AMR between host species, have not been identified thus far.

Comparative genomic analysis of bacterial populations from multiple hosts has revealed signatures of host-adaptation in bacterial genomes(10). The emergence of large-scale bacterial genome-wide association studies (GWAS) allowed for the detection of genes or genomic variants that are associated with resistance, pathogenicity, and host adaptive traits(11-13). Here, we have applied population-based bacterial GWAS to identify host-associated genomic determinants in a diverse panel of 1,198 E. coli isolates, irrespective of their AMR pattern. Isolates were recovered from five different host species, including healthy and diseased individuals from four different countries in two continents over 16 years. The pan-genome was 
analyzed for specific host association followed by a $k$-mer based bacterial GWAS approach to identify host-specific genomic determinants and their potential role in host-adaptation.

\section{Material and Methods}

\section{a) Sampling strategy}

107 A panel of 1213 E. coli isolates from four countries (Germany, UK, Spain, and Vietnam), 108 obtained from five host species (human, pig, cattle, chicken, and wild boar) during three time 109 periods (2003-2007, 2008-2012 and 2013-2018) from both healthy and diseased hosts were 110 selected randomly from existing strain collections and newly collected isolates. Out of 120

111 possible strata (defined as a unique combination of country, host, time-period, and host health

112 status), 42 strata contained isolates. We included all isolates available per stratum if there were 113 less than 30 isolates and performed a random selection of up to a maximum of 30 isolates if 114 more were available. Potentially duplicate isolates that were part of an outbreak, isolated at a 115 single location within a short timeframe, or from a single farm or a single individual were excluded. Only one isolate per individual was included in the analyses. Isolates included per

117 stratum are shown in Table S1.

\section{b) DNA extraction and sequencing}

119 The DNA of the E. coli isolates from Germany was extracted using the QIAamp DNA Mini 120 Kit (Qiagen) following the manufacturer's instructions. The DNA concentration was evaluated

121 fluorometrically by using Qubit ${ }^{\mathrm{TM}} 2.0$ fluorometer (Invitrogen, USA) and the associated 122 Qubit $^{\mathrm{TM}}$ dsDNA HS Assay Kit (0.2-100ng) and Qubit ${ }^{\mathrm{TM}}$ BR Assay Kit (2-1000ng), respectively. The libraries were generated using Nextera DNA library preparation (Illumina,

124 https://www.illumina.com). The sequencing was performed using the Illumina MiSeq and 125 HiSeq systems, generating $2 \times 250 \mathrm{bp}$ and $2 \times 150 \mathrm{bp}$ reads, respectively. 
126 The DNA of the E. coli isolates from the UK was purified using a Promega DNA Wizard $\AA$

127 genomic purification kit and quantified using Nanodrop. Libraries were generated using

128 Nextera XT technology (Illumina), and DNA sequencing of isolates was performed at the

129 Animal and Plant Health Agency (APHA, Surrey, UK, https://www.gov.uk/government/-

130 organisations/animal-and-plant-healthagency) using an Illumina MiSeq system generating $2 \times$

131150 bp reads.

132 For E. coli isolates from Spain, DNA was extracted using the DNA blood and tissue Qiagen

133 kit according to the manufacturer's instruction. The total amount of DNA was quantified using

134 a Qubit fluorometer and frozen at $-20^{\circ} \mathrm{C}$ until further analysis. Libraries were prepared using

135 Nextera XT DNA Library preparation (Illumina), and DNA samples were sequenced using a

136 MiSeq platform $(2 \times 300$ cycle V3 Kit $)$.

137 The DNA of the E. coli isolates from Vietnam was extracted using the Wizard Genomic DNA

138 purification kit (Promega, Madison, WI, USA) following the manufacturer's instructions. The

139 concentration of the DNA was measured fluorometrically by using picogreen (Invitrogen). The

140 sequencing was performed using an Illumina HiSeq 4000 system, which generates $2 \times 150 \mathrm{bp}$

141 reads.

\section{c) Quality control}

143 Adapter sequences were removed from raw reads using flexbar v3.0.3(14,15) with trimming

144 mode (-ae) ANY. Low-quality bases within raw reads (Phred score value $<20)$ were trimmed

145 using a sliding window approach (-q WIN). FastQC v0.11.7(16) and MultiQC v1.6(17) were

146 used for quality control before and after processing steps.

\section{7 d) Genome assembly and annotation}

148 Adapter-trimmed reads were assembled using SPAdes v3.13.1(18) using read correction.

149 Scaffolds smaller than 500bp were discarded. QUAST v5.0.0(19) was used to assess assembly 
quality using default parameters. Draft assemblies were excluded if the N50 was below an aribrary value of $30 \mathrm{kbp}$ or consisted of more than 900 contigs. Draft genomes were annotated using prokka v1.13(20) with a genus-specific blast for Escherichia. Phylogroups were predicted using ClermonTyper v1.4.1(21), and sequence types (STs) of the isolates were

154 identified in silico using the Achtman seven gene MLST scheme using mlst (https://github.com/tseemann/mlst).

\section{e) Pan-genome and phylogenetic analysis}

157 Roary v3.12.0(22) was used to define the pan-genome of the population, using paralog splitting. The core genes were aligned using prank(23) on default parameters. The core gene alignment was used to construct the phylogenetic tree using RaxML 8.2.4(24) with 100

161 of rate heterogeneity and Lewis ascertainment bias correction(25). The core gene phylogeny

162 was corrected for recombination using ClonalFrameML(26) using default parameters.

163 Phylogenetic Clusters (or BAPS clusters) within the dataset were defined using hierBAPS $(27,28)$ based on the core gene alignment. The accessory gene clustering was performed using package Rtsne v0.15(29,30) with 5000 iterations and perplexity 15 in $\mathrm{R}$ v3.6.1. iTOL(31) and Microreact(32) were used to visualize the population structure in the

167 context of available metadata. The function chisq.test from the MASS library(33) (v7.3-51.1) was used in $\mathrm{R}(34)$ (v3.5.2) to perform $\mathrm{X}^{2}$-tests of independence between phylogenetic clusters and host species. Tests were carried out on the full dataset (14 phylogenetic clusters vs. five hosts and nine phylogroups vs. five host species).

\section{f) Genome-wide association study (GWAS)}

172 We excluded the wild boar E. coli isolates from the GWAS analysis, because of their low number ( $\mathrm{n}=29)$. GWAS was performed to screen $k$-mers for associations with their host (pig, 
human, chicken, and cattle). Assemblies were shredded into k-mers of 9-100 bases using FSM-

175 lite (https://github.com/nvalimak/fsm-lite). The association between $k$-mers and host

176 phenotype was carried out using Fast-LMM linear mixed model implemented in pyseer(35)

177 using a pairwise similarity matrix derived from the phylogenetic tree as population correction.

178 A GWAS analysis was carried out for each host (pig, human, chicken, and cattle). To reduce

179 false-positive associations, isolates from the host of interest were compared with an equal

180 number of isolates from each of the other hosts, designated control isolates. This analysis was

181 repeated 100 times per host of interest by selecting the control strains from other hosts per

182 iteration(36). The selection of control isolates was random and with replacement except for

183 stratification by phylogenetic clusters to minimize phylogenetic bias. The statistical significance threshold was estimated based on the number of unique $k$-mers patterns for each run(35). K-mers, which were significantly associated with $90 \%$ of the runs per host, were retained and mapped to reference genomes (Table S2) using a fastmap algorithm in bwa(35,37).

187 An arbitrary cut-off of a minimum of 10 k-mers mapped per gene was chosen for further 188 analysis to reduce false-positives. In silico characterization and gene ontology (GO) assignment was performed using Blast2GO(38), and Clusters of Orthologous Groups (COGs) were assigned using CD-search $(39,40)$.

\section{g) Prevalence of a human-associated nan gene cluster}

192 All available E. coli genome assemblies in NCBI RefSeq were downloaded on Nov 29 ${ }^{\text {th }}, 2019$, using NCBI-genome-download (https:/github.com/kblin/ncbi-genome-download). Using a custom ABRicate (https://github.com/tseemann/abricate) database, consisting of the nine genes of the novel human-associated nan gene cluster, all downloaded genomes $(n=17994)$ were scanned. STs for all the genomes were assigned as described above. 


\section{h) Construction of mutants and phenotypic experiments}

198 Mutants $\triangle$ nan-9 $\left(\mathrm{Amp}^{\mathrm{R}}\right)$ and $\triangle n a n R A T E K$ of extra-intestinal pathogenic E. coli (ExPEC) strain

199 IMT12185 (ST131; RKI 20-00501; $\mathrm{Amp}^{\mathrm{R}}$ ) were constructed using the Datsenko-Wanner

200 method(41). The genomic DNA of the wild-type and the mutant strains was isolated using a

201 QIAamp DNA Mini Kit (QIAGEN). Libraries were prepared using the Nextera XT DNA

202 Library preparation kit (Illumina), and MinION one-dimensional (1D) libraries were

203 constructed using the SQK-RBK004 kit (Nanopore technologies, Oxford, UK) and loaded according to the manufacturer's instructions onto an R9.4 flow cell. MinIon sequencing data were collected for $48 \mathrm{~h}$ and the paired-end Illumina sequencing was performed using MiSeq.

with default parameters to complete both strains' genomes. The absence of the desired genes was confirmed based on the assembly followed by annotation using prokka v1.13(20).

209 Carbon utilization and chemical sensitivity of the deletion mutants and their parental strain

210 were tested using a Biolog Phenotypic Array system, using the PM1 MicroPlate and the Gen

211 III MicroPlate according to the manufacturer's instructions.

\section{2 i) Growth curve analysis}

213 E. coli strains were grown at $37^{\circ} \mathrm{C}$ aerobically in lysogeny broth (LB) $(10 \mathrm{~g} / 1$ tryptone, $5 \mathrm{~g} / 1$ 214 yeast extract, $5 \mathrm{~g} / \mathrm{l} \mathrm{NaCl}, \mathrm{pH} 7.5$ ) or in minimal medium (MM). $\mathrm{MM}$ is $\mathrm{M} 9$ mineral medium $215\left(33.7 \mathrm{mM} \mathrm{Na}_{2} \mathrm{HPO}_{4}, 22.0 \mathrm{mM} \mathrm{KH}_{2} \mathrm{PO}_{4}, 8.55 \mathrm{mM} \mathrm{NaCl}, 9.35 \mathrm{mM} \mathrm{NH} 4 \mathrm{Cl}\right)$ supplemented with

$2162 \mathrm{mM} \mathrm{MgSO}_{4}$ and $0.1 \mathrm{mM} \mathrm{CaCl}_{2}$. As carbon and energy source, either $27.8 \mathrm{mM}[0.5 \% \mathrm{w} / \mathrm{v}]$ 217 glucose, $6.47 \mathrm{mM}[0.2 \% \mathrm{w} / \mathrm{v}]$ 5-N-acetyl neuraminic acid (Neu5Ac), or $6.15 \mathrm{mM}$ [0.1\% w/v]

218 N-glycolylneuraminic acid (Neu5Gc) (all purchased from Sigma-Aldrich, Taufkirchen, 219 Germany) was added. If appropriate, the following antibiotics were used: ampicillin sodium salt $(150 \mu \mathrm{g} / \mathrm{ml})$ or kanamycin $(50 \mu \mathrm{g} / \mathrm{ml})$. For solid media, 1.5\% agar (w/v) was added. For

221 all growth experiments, bacterial strains were grown in LB medium overnight at $37^{\circ} \mathrm{C}$, washed 
222 twice in PBS and then adjusted to an optical density at $600 \mathrm{~nm}\left(\mathrm{OD}_{600}\right)$ of 0.005 in the desired

223 liquid growth medium, or streaked on agar plates. Growth curves were obtained from bacterial

224 cultures incubated at $37^{\circ} \mathrm{C}$ with gentle agitation in 96-well microtitre plates containing $200 \mu 1$

225 medium. The $\mathrm{OD}_{600}$ was measured by an automatic reader (Epoch2T; BioTek, Bad

226 Friedrichshall, Germany) at appropriate time intervals as indicated. 


\section{Results}

\section{Data collection}

230 After WGS quality control, 14 isolates were excluded because of poor quality sequences. One

231 additional isolate was excluded since this isolate was identified as Escherichia marmotae

232 (formerly cryptic clade V)(43,44), a species commonly mistaken for E. coli. Our final collection comprised 1198 E. coli whole-genome sequences with metadata (Table S1), which also contained 8 cryptic clade I isolates, which were included as E. coli based on the recommended species cut-off of $95-96 \%$ average nucleotide identity(43). Our collection consisted of $22.1 \%(n=265)$ cattle, $28.1 \%(n=337)$ chicken, $27.3 \%(n=327)$ human, $20.3 \%$ $(n=240)$ pigs and $2.4 \%(n=29)$ wild boar isolates (Fig. S1A). Fifty-one percent $(n=612), 19.4 \%$ $(n=233), 14.5 \%(n=174)$ and $14.9 \%(n=179)$ of these isolates were from Germany, Spain, the UK, and Vietnam, respectively (Fig. S1A). Chicken isolates were from all four countries, human isolates from Germany, the UK and Vietnam, pig isolates from Germany, Spain and Vietnam, cattle isolates from Germany and Spain and only Spain provided wild boar isolates. In total, $35.5 \%(\mathrm{n}=426)$ of the isolates were from hosts with reported disease, whereas $62.0 \%$ $(n=743)$ were from hosts without reported disease, while host health status was unknown for the wild boar isolates $(2.4 \%, \mathrm{n}=29)$. Of the 1198 isolates analyzed, 1140 were grouped into 358 different STs, and 58 could not be assigned to any known ST. The population structure of the collection closely resembles that of the ECOR collection(45), indicating that it represents most

247 of the known diversity of E. coli sensu stricto (Fig. S2).

\section{Pan-genome analysis}

249 The pan-genome of the 1198 E. coli isolates consisted of 77130 genes, of which 1956 genes

250 belonged to the core genome (i.e., present in at least $99 \%$ of the isolates). The population 
defined using Bayesian analysis of population structure (BAPS), which assigns isolates to phylogroups D ( $n=97,8.09 \%), E(n=55,4.59 \%), G(n=49,4.09 \%), F(n=35,2.92 \%), C(n=60$, $5.0 \%)$, and clade I ( $\mathrm{n}=8,0.6 \%)$. A comparison of phylogenetic clusters, phylogroups, country, host, and a maximum likelihood (ML) tree based on 110920 core-genome SNPs is shown in

Fig 1 . The $\chi^{2}$-tests for independence revealed a positive correlation between host status and phylogenetic clusters (at $\left.p<2.26 \mathrm{e}^{-16}, \mathrm{df}=52\right)$ and between phylogroups and hosts $\left(p<2.2 \mathrm{e}^{-16}\right.$, $\mathrm{df}=32$ ). This indicates that specific phylogenetic clusters (Fig. S1 B\&C) and phylogroups, such as B1 (cattle), A (pig), B2 (human and chicken), and G (chicken) were enriched within different hosts in our collection (Fig. S1D).

263 Clustering of isolates based both on core gene alignment and on accessory gene profile appeared to be correlated with phylogroups. The interactive visualization of data is also available on Microreact (https://microreact.org/project/ouDOdcFxc). A minimum spanning tree was built on the allelic profiles of $358(\mathrm{n}=1,140$ isolates) known STs and 58 isolates belonging to unknown STs using GrapeTree(46) along with the host distribution (Fig. S3). Several sequence types, of which at least ten isolates were available, appeared to be linked with certain host species. ST33 ( $n=10 / 10,10$ human isolates out of all 10 isolates), ST73 (n=11/17), ST131 $(n=37 / 42)$ and ST1193 $(n=12 / 12)$ were associated with a human host. ST131 was also found in chickens $(n=4 / 42)$ and pigs $(n=1 / 42)$ in this collection. ST23 $(n=18 / 22)$, ST95 $(n=25 / 31)$, ST115 $(n=11 / 11)$, ST117 $(n=30 / 33)$, ST140 $(n=19 / 20)$ and ST752 $(n=29 / 30)$ were associated with the chicken host. 


\section{GWAS}

278 The genome-wide association analysis was performed on $1169 \mathrm{E}$. coli isolates from cattle, 279 chickens, humans, and pigs. The 29 wild boar isolates were excluded because of their small

280 group size. Genome-wide association analysis revealed the positive association $(\beta>0)$ of

$28127,854,16,164$, and 69,307 k-mers with $E$. coli isolates from humans, cattle, and chickens at a

282 likelihood ratio test $p$-value less than $1.87 \times 10^{-9}, 2.16 \times 10^{-9}$, and $1.9 \times 10^{-9}$ respectively (reported

283 as "Irt-pvalue"). There were no k-mers significantly associated with the pig host. The significant $k$-mers accounted for 426, 179, and 915 bacterial genes associated with isolation

285 from human, cattle, and chicken hosts, respectively (Fig 2 and Table S3). An arbitrary cut-off

286 of at least $10 \mathrm{k}$-mers mapped per gene was chosen to select genes for in silico functional 287 characterization as well as COG assignment using Blast2GO(38) (Table S4) and CD$288 \operatorname{search}(39,40)$ (Fig. S4).

\section{Association of novel nan genes with human host}

290 GWAS revealed a strong association of nine contiguous genes, assigned to the group of nan 291 genes with the human host (Fig 2b). Seven of these genes were annotated in silico as nan genes 292 (Fig 3a) and the remaining two genes were annotated as being similar to axeAl of Prevotella ruminicola ATCC 19189 (Uniprot accession D5EV35). However, the amino acid sequences of the products of these axeA1-like genes only shared 19-20\% similarity with AxeA1. Further investigation with EggNOG and CD search revealed an acetylesterase/lipase-encoding region (COG0657) in both genes and confirmed nan gene annotations. Previous evidence and the genomic location (i.e., between the nan genes; Fig 3a) suggest that these genes encode potential acetylesterases and may be analogous to sialyl esterases (NanS)(47). Hence, these nine novel nan genes are collectively termed "human-associated nan gene cluster (nan-9)" (Fig 3a). 
301 Distinct nan genes are present in E. coli and are also known as the sialoregulon (nanRATEK-

$302 y h c H$, nanXY $[y j h B C]$, and nanCMS; Fig 3a)(48). The sialoregulon is known to be involved in

303 metabolism of sialic acids(49-51), a diverse group of nine-carbon sugars, abundant in the

304 glycocalyx of many animal tissues $(52,53)$. Sialic acids present on mucin proteins in the human

305 gut are an essential energy source for many intestinal bacteria(54). The proteins encoded by

306 the seven genes of nan-9 (i.e. nanAKTCMRS) share 45-64\% similarity with the corresponding

307 nan genes of the sialoregulon in E. coli or the recently described phage-encoded nanS-p genes

308 of enterohemorrhagic E. coli(55). Both the human-associated nan gene cluster and the

309 sialoregulon are located on the bacterial chromosome. The human-associated nan gene cluster

310 was found in $7 \%$ of our isolate collection, whereas the genes comprising the sialoregulon were

311 more common. In our collection, nan $X Y$ was identified in $\sim 15 \%$ of isolates, nanCMS in $\sim 93 \%$

312 of isolates, whilst nanRATEKyhcH was found in almost all $(>99 \%)$ isolates.

314 The nan-9 cluster was detected in 86 isolates, mainly from phylogroups B2 and D (Fig 3b) and

315 predominantly in isolates belonging to ST131, ST73, and ST69, both in our collection as well

316 as across 17,994 RefSeq E. coli genomes (Fig 3c). The order and orientation of genes in the

317 human-associated nan gene cluster were found to be identical in 82 out of 86 isolates (Fig. S5).

318 In 63 isolates, insertion sequence (IS) 682 was found upstream, and in 23 isolates, IS2 was

319 found downstream of this novel gene cluster (Fig. S5).

320

321 To further explore the function of the human-associated nan-9 gene cluster, the entire cluster

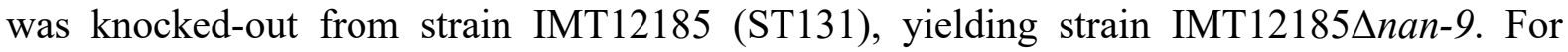
comparison, an additional mutant, which lacked the nanRATEK locus from the sialoregulon

324 (IMT12185 $\triangle$ nanRATEK) was constructed from wild-type IMT12185. Correct gene deletion in 
chemical sensitivity were observed between wild-type strain IMT12185 and its mutant

IMT12185 4 nan-9 in Biolog phenotyping array experiments (PM1 and Gen III MicroPlates).

Deletion mutant IMT121854nan-9 was grown in MM with $0.2 \%$ 5-N-acetylneuraminic acid

330 (Neu5Ac) or with $0.1 \%$ N-glycolylneuraminic acid (Neu5Gc) as sole carbon and energy

IMT12185 $\Delta$ nan-9 grew to a maximal OD $_{600}$ of 1.34 comparable to that of parental strain

IMT12185 $\left(\mathrm{OD}_{600}=1.37\right)$. However, the mutant exhibited a delayed growth start of maximal $\mathrm{OD}_{600}(1.31)$ in comparison with strain IMT12185 $\left(\mathrm{OD}_{600}=1.43\right)($ Fig $4 b)$. Both

338 Neu5Ac and Neu5Gc are degraded by the enzymatic activities of the enzymes NanRATEK, of which four, namely NanRATK, are encoded by redundant genes located on the determinants nanRATEK and nan-9. Deletion mutant IMT12185 $\operatorname{nanRATEK}$ was unable to grow with

341 Neu5Ac (Fig 4c), demonstrating that nan-9 alone is not sufficient for sialic acid degradation,

342 probably due to a lack of nanE in the nan-9 gene cluster. To exclude a pleiotropic effect of the

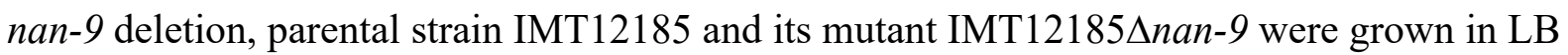

344 medium. No significant difference was observed between the two growth curves (Fig 4d).

345 These data demonstrate that the nan-9 determinant of strain IMT12185 is biologically

346 functional and contributes to the degradation of the sialic acids Neu5Ac and Neu5Gc. 
Other genes associated with the human host

350 Several other genes associated with the human host were identified in the GWAS analysis, such as the sat gene encoding a serine protease autotransporter vacuolating toxin (Fig $2 b)(56)$. detected in isolates belonging to specific lineages such as ST131, ST1193, and ST73 (Table S5). In addition, we found an association with two distinct homologs of the macB gene that encodes an $\mathrm{ABC}$ transporter(57) and is involved in many diverse processes, such as resistance to macrolides(58), lipoprotein trafficking(59), and cell division(60).

\section{Association of distinct Omptins with the cattle and chicken hosts}

359 We detected homologs of the ompT (encoding outer-membrane protease VII) gene, a member of the omptin family of proteases, in our dataset (Fig 2a \& Fig 2c). Two homologs, ompP

361 (UniProt accession P34210, sharing 70\% amino acid identity with OmpT) and arlC (also referred to as ompTp, UniProt accession Q3L7I1, sharing 74\% amino acid identity with OmpT), were found to be associated with the cattle and chicken hosts, respectively (Fig 5). In our collection, ompP was predominant in phylogroup B1 $(\mathrm{n}=68)$, whereas $\operatorname{arlC}$ was found in distinct phylogroups (such as B2, B1 and G) (Fig 5) and in isolates belonging to ST95 and RefSeq (Table S6). Previous studies have reported an increased prevalence of arlC (erroneously reported there as ompT) in a cluster of uropathogenic E. coli (UPEC) and avian pathogenic E. coli (APEC) classified as ST95(61). Notably, arlC is associated with increased degradation of antimicrobial peptides (AMPs) in UPEC isolates(62). OmpP is also able to degrade AMPs and displays a AMP cleavage specificity different from that of OmpT(63). 
bioRxiv preprint doi: https://doi.org/10.1101/2022.02.08.479532; this version posted February $9,2022$. The copyright holder for this preprint (which was not certified by peer review) is the author/funder, who has granted bioRxiv a license to display the preprint in perpetuity. It is made available under aCC-BY-NC 4.0 International license.

\section{Association of genes involved in metal acquisition with the chicken host}

374 GWAS analysis revealed an association of the iroBCDEN gene cluster (C) with the chicken 375 host, but not with other host species included in this study. The prevalence of the iro gene 376 cluster was $24.3 \%(n=291 / 1198)$ in our collection, of which $61.5 \%(n=179 / 291)$ were from the

377 chicken host. The gene cluster was found in different STs and with higher prevalence in STs 378 such as ST117, ST95, ST23, and ST140 (Table S7). The chromosomal iroBCDEN gene cluster

379 was first described in Salmonella enterica and is involved in uptake of catecholate-type 380 siderophores, high-affinity iron-chelating molecules contributing to bacterial survival during

381 infection by sequestering iron(64). In E. coli, this gene cluster has mainly been described in 382 uropathogenic (UPEC) and avian pathogenic E. coli (APEC) and is regarded as a virulence 383 factor(65). The cluster has been reported on a chromosomal pathogenicity island, although in

384 ExPEC, the cluster can also be located on ColV or ColBM virulence plasmids(66,67). In addition, homologs of genes involved in zinc catabolism $(z n u B)$ and iron metabolism (fes) were found to be associated with the chicken host (Fig 2c). 


\section{Discussion}

388

Escherichia coli can colonize many different ecological niches in a diverse range of host species, ranging from a commensal lifestyle to intra- or extra-intestinal infections. Presence of certain adhesin and other virulence-associated genes is well known to correlate with the relative ability of E. coli strains to colonize the intestinal tract of certain hosts (e.g., ecp for humans(68), F9 fimbriae and H7 flagellae for cattle $(69,70)$ or Stg fimbriae for chickens(71)). Variations in host adaptation levels and their molecular basis in E. coli strains presumptively realizing a commensal-like lifestyle in the reservoir host are rarely described and poorly understood as of yet(72). Commensal E. coli strains may be carriers of AMR and a source of mobile genetic elements conferring AMR to other bacteria including pathogenic strains in a shared microbiome, e.g. in the intestinal tract of animals including humans. We therefore collated an extensive and diverse dataset to identify genetic determinants of E. coli host adaptation. We observed significant enrichment of specific hosts within some phylogroups and STs in our collection. Furthermore, we unveiled correlations between the likelihood of genetically related isolates having been isolated from a certain host with the possession of distinctive genetic traits. Some of these traits, e.g. the iroBCDEN gene cluster, have been linked to E. coli and Salmonella virulence before, while others, in particular the human-associated nan gene cluster, are novel traits and have not been implicated in the infection and colonization process of $E$. coli. Of note, the latter gene cluster encodes for metabolic properties which have received little attention in bacterial infectious disease research. Specific metabolic properties have been linked to the relative ability of Shiga toxin-encoding E. coli (STEC) to asymptomatically colonize cattle, their reservoir host(73). Unraveling the nutrient and energy flows in the complex interplay of intestinal bacteria, the surrounding microbiome and the host may open novel avenues to control the persistence and transmission of pathogenic and/or antimicrobial resistant bacteria(74). 
413 We employed a k-mer based bacterial GWAS, applied in previous studies to associate multiple

414 types of genetic variation with phenotypes $(75,76)$. In our study, we were able to associate a

415 phenotype (i.e., isolates obtained from a certain host species) with the presence of specific

416 genes, but not with sequence variation at the level of single nucleotide polymorphisms between

417 genes. This lack of associations found at the SNP level could possibly be explained by the fact

418 that through our filtering approach to prevent false positive hits, we might have excluded $k$ -

419 mers that captured host-associated SNP variation. Secondly, it might be possible that since

420 E. coli is genetically diverse, host-associated SNP variation is challenging to capture between

421 unrelated strains. Finally, the absence of host-associated SNPs might be a biological observation, indicating that colonization of particular hosts is determined by gene presence or absence rather than minimal genetic variation within genetic elements. However, we were able example, carriage of the salmochelin operon encoded by iroBCDEN and involved in iron metabolism was previously identified as associated with increased ability of E. coli strains to colonize chickens $(65,77)$.

In addition to iroBCDEN, we found an association of omptin proteins (OmpP and ArlC) with chickens and cattle as hosts, respectively. Earlier studies using UPEC strains had demonstrated that these proteins are associated with cleavage and inactivation of cationic antimicrobial peptides (AMPs)(62). Because AMPs are secreted as part of the host's innate immune response(78-80), these proteins may play a vital role in colonization. AMPs are also increasingly used as alternatives to antimicrobial agents in animal farming(81-83), further investigation into the contribution of these Omp variants to host colonization as well as to resistance to exogenous AMPs is warranted. 
437 We did not identify any significant associations of $k$-mers with the pig host. Bacterial

438 colonization of the porcine intestine by edema-disease $E$. coli (EDEC) is mediated by the ability

439 of these bacteria to adhere to villous epithelial cells via their cytoadhesive F18 fimbriae(84).

440 The expression of receptors for these fimbriae on the apical enterocyte surface is inherited as a

441 dominant trait among pigs and determines susceptibility to diseases caused by F18-fimbriated

442 pathogenic E. coli(85). Enterotoxigenic E. coli (ETEC) express F4 or F5 fimbriae with similar

443 consequences(86). However, we found only three, four and six isolates harbouring genes for

444 F4, F5 and F18 fimbriae, respectively. Thus, we might not have had all E. coli pathovars associated with pig host sufficiently present in our collection, although we did observe an association between phylogroup A and pig colonization. An alternative reason might be that the association between phylogroup A and pig colonization complicated the identification of statistically significant $k$-mers. GWAS corrects for population structure, which means that if there is a strong association between lineage and phenotype, the genes harbored by that lineage will not be reported as having a strong association with the phenotype under study(87).

We identified a novel human host-associated nan gene cluster, distinct from the previously reported sialic acid (Sia) metabolic operon (nanRATEK-yhcH, nanXY, and nanCMS)(48). This novel cluster is conserved and abundant in ExPEC lineages, such as ST131, ST73, and ST69. The gene cluster is flanked by insertion sequences which might play a role in the horizontal exchange between different $E$. coli lineages. Knock-out in vitro studies indicated that this novel nan-9 gene cluster contributes to catabolism of the sialic acids Neu5Ac and Neu5Gc, although it cannot replace the function of the nanRATEK locus which is abundant in E. coli. Hence, we hypothesize that E. coli harboring the nan-9 gene cluster have an evolutionary advantage through either more efficient access to sialic acids or through access to more diverse sialic acids. The genes annotated as acetylxylan esterases are expected to represent novel sialyl 
esterases, as known sialyl esterases (nanS variants) have previously been mistaken for acetylxylan esterases(47). Additional sialyl esterases - possibly with alternative deacetylation specificity - might provide a more efficient catabolism of acetylated sialic acids. Future studies should investigate the role of the human-associated nan-9 gene cluster in the catabolism of differentially acetylated sialic acids and their relevance for the human host.

467

Approximately one-third of the isolates in our dataset were obtained from diseased hosts, while the remaining isolates were from healthy hosts. Many of the isolates in our dataset that originate from healthy hosts belong to ExPEC lineages which are typically considered to be pathogenic. In fact, the locus most strongly associated with the human host, the nan-9 gene cluster, is abundant in ExPEC lineages. This does not necessarily mean that the nan-9 gene cluster is associated with pathogenicity. In fact, this observation primarily supports the notion that these pathogenic E. coli are highly efficient colonizers of the human intestine(72). Based on our results, we hypothesize that the human-associated nan-9 gene cluster is one of the factors driving the adaptation of ExPEC to the human intestine.

Finally, we observed an association between the sat gene and human host colonization. Sat contributes to the pathogenicity of $E$. coli in the urinary tract(56). The high prevalence of sat in previously studied E. coli isolates from the feces of healthy individuals suggests it may not act as a virulence factor in the human gut(88). However, in our isolate collection, the sat gene was found in E. coli strains belonging to phylogroups A, B2, D, and F, which had been isolated from both healthy and diseased hosts (Table S5). Understanding the role of Sat in the colonization and adaptation of $E$. coli in healthy humans warrants further investigation. 


\section{Conclusion}

488 Our study identified several distinct genetic determinants that may influence E. coli adaptation

490 they aid the better understanding of the potential outcome of transmission events of E. coli

491 between host species. This is particularly relevant for the control of the spread of antimicrobial

492 resistant commensal and zoonotic E. coli strains within and across human and animal 493 populations. The data generated here can also be used in risk analysis and for diagnostic and monitoring purposes. More importantly, our study identified biological processes, including sialic acid catabolism, that should be investigated in more detail to better understand $E$. coli

496 host adaptation.

497

498 Data availability:

499 The raw-reads of the 1090 E. coli isolates sequenced in this study were submitted to NCBI 500 SRA with the Bioproject accession number PRJNA739205 and the SRA accession of 108 501 isolates, that were taken from other studies, were provided in supplement table S1.

\section{Acknowledgements:}

503 The HECTOR research project was supported under the framework of the JPIAMR - Joint

504 Programming Initiative on Antimicrobial Resistance - through the $3^{\text {rd }}$ joint call, thanks to the generous

505 funding by the Netherlands Organisation for Health Research and Development (ZonMw, grant number 506 547001012), the Federal Ministry of Education and Research (BMBF/DLR grant numbers 01KI1703A, 507 01KI1703C and 01KI1703B), the State Research Agency (AEI) of the Ministry of Science, Innovation 508 and Universities (MINECO, grant number PCIN-2016-096), and the Medical Research Council (MRC, 509 grant number MR/R002762/1).

\section{Competing Interests:}

511 The authors declare no competing interests. 


\section{References:}

513 1. Alteri CJ, Mobley HLT. Escherichia coli physiology and metabolism dictates adaptation to diverse host

microenvironments. Vol. 15, Current Opinion in Microbiology. 2012

2. Ewers C, Bethe A, Semmler T, Guenther S, Wieler LH. Extended-spectrum $\beta$-lactamase-producing and AmpC-producing Escherichia coli from livestock and companion animals, and their putative impact on public health: A global perspective. Clinical Microbiology and Infection. 2012.

3. Bonnet R, Beyrouthy R, Haenni M, Nicolas-Chanoine M-H, Dalmasso G, Madec J-Y. Host Colonization as a Major Evolutionary Force Favoring the Diversity and the Emergence of the Worldwide Multidrug-Resistant Escherichia coli ST131 . MBio. 2021;12(4).

4. Lopatkin AJ, Meredith HR, Srimani JK, Pfeiffer C, Durrett R, You L. Persistence and reversal of plasmid-mediated antibiotic resistance. Nat Commun. 2017;8(1).

5. Pitout JDD, DeVinney R. Escherichia coli ST131: A multidrug-resistant clone primed for global domination. F1000Research. 2017.

6. Nicolas-Chanoine MH, Bertrand X, Madec JY. Escherichia coli st131, an intriguing clonal group. Clin Microbiol Rev. 2014;

7. Skurnik D, Clermont O, Guillard T, Launay A, Danilchanka O, Pons S, et al. Emergence of antimicrobial-resistant Escherichia coli of animal origin spreading in humans. Mol Biol Evol. 2016;

8. Riley LW. Pandemic lineages of extraintestinal pathogenic Escherichia coli. Vol. 20, Clinical Microbiology and Infection. 2014.

9. Cohen E, Azriel S, Austeri O, Gal A, Zitronblat C, Mikhlin S, et al. Pathoadaptation of the passerineassociated Salmonella enterica serovar Typhimurium lineage to the avian host. PLoS Pathog. 2021;17(3).

10. Toft C, Andersson SGE. Evolutionary microbial genomics: Insights into bacterial host adaptation. Nature Reviews Genetics. 2010.

11. Sheppard SK, Didelot X, Meric G, Torralbo A, Jolley KA, Kelly DJ, et al. Genome-wide association study identifies vitamin B5 biosynthesis as a host specificity factor in Campylobacter. Proc Natl Acad Sci U S A. 2013;

12. Mageiros L, Méric G, Bayliss SC, Pensar J, Pascoe B, Mourkas E, et al. Genome evolution and the emergence of pathogenicity in avian Escherichia coli. Nat Commun. 2021;12(1).

13. Salipante SJ, Roach DJ, Kitzman JO, Snyder MW, Stackhouse B, Butler-Wu SM, et al. Large-scale genomic sequencing of extraintestinal pathogenic Escherichia coli strains. Genome Res. 2015;

14. Dodt M, Roehr JT, Ahmed R, Dieterich C. FLEXBAR-flexible barcode and adapter processing for nextgeneration sequencing platforms. Biology (Basel). 2012;

15. Roehr JT, Dieterich C, Reinert K. Flexbar 3.0 - SIMD and multicore parallelization. Bioinformatics. 2017;

16. Andrews S, Krueger F, Seconds-Pichon A, Biggins F, Wingett S. FastQC. A quality control tool for high throughput sequence data. Babraham Bioinformatics. Babraham Institute. 2015.

17. Ewels P, Magnusson M, Lundin S, Käller M. MultiQC: Summarize analysis results for multiple tools and samples in a single report. Bioinformatics. 2016;

18. Bankevich A, Nurk S, Antipov D, Gurevich AA, Dvorkin M, Kulikov AS, et al. SPAdes: A new genome assembly algorithm and its applications to single-cell sequencing. J Comput Biol. 2012;

19. Gurevich A, Saveliev V, Vyahhi N, Tesler G. QUAST: Quality assessment tool for genome assemblies. Bioinformatics. 2013;

20. Seemann T. Prokka: Rapid prokaryotic genome annotation. Bioinformatics. 2014;

21. Beghain J, Bridier-Nahmias A, Nagard H Le, Denamur E, Clermont O. ClermonTyping: An easy-to-use and accurate in silico method for Escherichia genus strain phylotyping. Microb Genomics. 2018;

22. Page AJ, Cummins CA, Hunt M, Wong VK, Reuter S, Holden MTG, et al. Roary: Rapid large-scale prokaryote pan genome analysis. Bioinformatics. 2015;

23. Löytynoja A. Phylogeny-aware alignment with PRANK. Methods Mol Biol. 2014;

24. Stamatakis A. RAxML version 8: A tool for phylogenetic analysis and post-analysis of large phylogenies. Bioinformatics. 2014;

25. Lewis PO. A likelihood approach to estimating phylogeny from discrete morphological character data. Syst Biol. 2001;

26. Didelot X, Wilson DJ. ClonalFrameML: Efficient Inference of Recombination in Whole Bacterial Genomes. PLoS Comput Biol. 2015;

27. Cheng L, Connor TR, Sirén J, Aanensen DM, Corander J. Hierarchical and spatially explicit clustering of DNA sequences with BAPS software. Mol Biol Evol. 2013;

28. Tonkin-Hill G, Lees JA, Bentley SD, Frost SDW, Corander J. RhierBAPs: An R implementation of the population clustering algorithm hierbaps [version 1; referees: 2 approved]. Wellcome Open Res. 2018; 
29. Van Der Maaten LJP, Hinton GE. Visualizing high-dimensional data using t-sne. J Mach Learn Res. 2008;

30. Van Der Maaten L. Accelerating t-SNE using tree-based algorithms. J Mach Learn Res. 2015;

574 31. Letunic I, Bork P. Interactive Tree Of Life (iTOL): An online tool for phylogenetic tree display and annotation. Bioinformatics. 2007;

32. Argimón S, Abudahab K, Goater RJE, Fedosejev A, Bhai J, Glasner C, et al. Microreact: visualizing and sharing data for genomic epidemiology and phylogeography. Microb genomics. 2016;

33. Venables WN, Ripley BD. Modern Applied Statistics with S Fourth edition by. Vol. 53, World. 2002.

34. R Core Team (2020). R: A language and environment for statistical computing. R Foundation for Statistical Computing, Vienna, Austria. 2020.

35. Lees JA, Galardini M, Bentley SD, Weiser JN, Corander J. pyseer: A comprehensive tool for microbial pangenome-wide association studies. Bioinformatics. 2018;

36. Epping L, Walther B, Piro RM, Knüver MT, Huber C, Thürmer A, et al. Genome-wide insights into population structure and host specificity of Campylobacter jejuni. Sci Rep. 2021;11(1).

37. Li H, Durbin R. Fast and accurate short read alignment with Burrows-Wheeler transform. Bioinformatics. 2009;

38. Conesa A, Götz S, García-Gómez JM, Terol J, Talón M, Robles M. Blast2GO: A universal tool for annotation, visualization and analysis in functional genomics research. Bioinformatics. 2005;

39. Marchler-Bauer A, Bryant SH. CD-Search: Protein domain annotations on the fly. Nucleic Acids Res. 2004;

40. Lu S, Wang J, Chitsaz F, Derbyshire MK, Geer RC, Gonzales R, et al. CDD / SPARCLE : the conserved domain database in 2020. 2020;48(November 2019):265-8.

41. Datsenko KA, Wanner BL. One-step inactivation of chromosomal genes in Escherichia coli K-12 using PCR products. Proc Natl Acad Sci U S A. 2000;

42. Wick RR, Judd LM, Gorrie CL, Holt KE. Unicycler: Resolving bacterial genome assemblies from short and long sequencing reads. PLoS Comput Biol. 2017;

43. Chun J, Oren A, Ventosa A, Christensen H, Arahal DR, da Costa MS, et al. Proposed minimal standards for the use of genome data for the taxonomy of prokaryotes. Int J Syst Evol Microbiol. 2018;

44. Liu S, Jin D, Lan R, Wang Y, Meng Q, Dai H, et al. Escherichia marmotae sp. nov., isolated from faeces of Marmota himalayana. Int J Syst Evol Microbiol. 2015;

45. Ochman H, Selander RK. Standard reference strains of Escherichia coli from natural populations. J Bacteriol. 1984;

46. Zhou Z, Alikhan N, Sergeant MJ, Luhmann N, Vaz C, Francisco AP, et al. GrapeTree : visualization of core genomic relationships among 100,000 bacterial pathogens. 2018;1395-404.

47. Steenbergen SM, Jirik JL, Vimr ER. YjhS (NanS) is required for Escherichia coli to grow on 9-Oacetylated N-acetylneuraminic acid. J Bacteriol. 2009;

48. Kalivoda KA, Steenbergen SM, Vimr ER. Control of the Escherichia coli sialoregulon by transcriptional repressor NanR. J Bacteriol. 2013;

49. Vimr ER, Kalivoda KA, Deszo EL, Steenbergen SM. Diversity of Microbial Sialic Acid Metabolism. Microbiol Mol Biol Rev. 2004;

50. Vimr ER, Troy FA. Identification of an inducible catabolic system for sialic acids (nan) in Escherichia coli. J Bacteriol. 1985;

51. Bell A, Severi E, Lee M, Monaco S, Latousakis D, Angulo J, et al. Uncovering a novel molecular mechanism for scavenging sialic acids in bacteria. J Biol Chem. 2020;295(40).

52. Vimr ER. Unified Theory of Bacterial Sialometabolism: How and Why Bacteria Metabolize Host Sialic Acids. ISRN Microbiol. 2013;

53. Severi E, Hood DW, Thomas GH. Sialic acid utilization by bacterial pathogens. Microbiology. 2007.

54. Haines-menges BL, Whitaker WB, Lubin JB, Boyd EF. Host Sialic Acids: A Delicacy for the Pathogen with Discerning Taste. In: Metabolism and Bacterial Pathogenesis. 2015.

55. Saile N, Voigt A, Kessler S, Stressler T, Klumpp J, Fischer L, et al. Escherichia coli O157:H7 strain EDL933 harbors multiple functional prophage-associated genes necessary for the utilization of 5-Nacetyl-9-O-acetyl neuraminic acid as a growth substrate. Appl Environ Microbiol. 2016;82(19).

56. Guyer DM, Henderson IR, Nataro JP, Mobley HLT. Identification of Sat, an autotransporter toxin produced by uropathogenic Escherichia coli. Mol Microbiol. 2000;

57. Kobayashi N, Nishino K, Yamaguchi A. Novel macrolide-specific ABC-type efflux transporter in Escherichia coli. J Bacteriol. 2001;

58. Tikhonova EB, Devroy VK, Lau SY, Zgurskaya HI. Reconstitution of the Escherichia coli macrolide transporter: The periplasmic membrane fusion protein MacA stimulates the ATPase activity of MacB. Mol Microbiol. 2007;

59. Khwaja M, Ma Q, Saier MH. Topological analysis of integral membrane constituents of prokaryotic 
ABC efflux systems. Res Microbiol. 2005;

60. Yakushi T, Masuda K, Narita SI, Matsuyama SI, Tokuda H. A new ABC transporter mediating the detachment of lipid-modified proteins from membranes. Nat Cell Biol. 2000;

61. Johnson TJ, Wannemuehler Y, Johnson SJ, Stell AL, Doetkott C, Johnson JR, et al. Comparison of extraintestinal pathogenic Escherichia coli strains from human and avian sources reveals a mixed subset representing potential zoonotic pathogens. Appl Environ Microbiol. 2008;

62. Desloges I, Taylor JA, Leclerc JM, Brannon JR, Portt A, Spencer JD, et al. Identification and characterization of OmpT-like proteases in uropathogenic Escherichia coli clinical isolates. Microbiologyopen. 2019;

63. Hwang BY, Varadarajan N, Li H, Rodriguez S, Iverson BL, Georgiou G. Substrate specificity of the Escherichia coli outer membrane protease OmpP. J Bacteriol. 2007;

64. Ratledge C, Dover LG. Iron Metabolism in Pathogenic Bacteria. Annu Rev Microbiol. 2000;

65. Caza M, Lépine F, Milot S, Dozois CM. Specific roles of the iroBCDEN genes in virulence of an avian pathogenic Escherichia coli O78 strain and in production of salmochelins. Infect Immun. 2008;

66. Sorsa LJ, Dufke S, Heesemann J, Schubert S. Characterization of an iroBCDEN gene cluster on a transmissible plasmid of uropathogenic Escherichia coli: Evidence for horizontal transfer of a chromosomal virulence factor. Infect Immun. 2003;

67. Dobrindt U, Blum-Oehler G, Nagy G, Schneider G, Johann A, Gottschalk G, et al. Genetic structure and distribution of four pathogenicity islands (PAI I536 to PAI IV536) of uropathogenic Escherichia coli strain 536. Infect Immun. 2002;

68. Rendón MA, Saldaña Z, Erdem AL, Monteiro-Neto V, Vázquez A, Kaper JB, et al. Commensal and pathogenic Escherichia coli use a common pilus adherence factor for epithelial cell colonization. Proc Natl Acad Sci U S A. 2007;104(25).

69. Low AS, Dziva F, Torres AG, Martinez JL, Rosser T, Naylor S, et al. Cloning, expression, and characterization of fimbrial operon F9 from enterohemorrhagic Escherichia coli O157:H7. Infect Immun. 2006;74(4).

70. Mahajan A, Currie CG, Mackie S, Tree J, Mcateer S, Mckendrick I, et al. An investigation of the expression and adhesin function of $\mathrm{H} 7$ flagella in the interaction of Escherichia coli O157: H7 with bovine intestinal epithelium. Cell Microbiol. 2009;11(1).

71. Lymberopoulos MH, Houle S, Daigle F, Léveillé S, Brée A, Moulin-Schouleur M, et al. Characterization of Stg fimbriae from an avian pathogenic Escherichia coli O78:K80 Strain and assessment of their contribution to colonization of the chicken respiratory tract. J Bacteriol. 2006;188(18).

72. Tenaillon O, Skurnik D, Picard B, Denamur E. The population genetics of commensal Escherichia coli. Nature Reviews Microbiology. 2010.

73. Barth SA, Weber M, Schaufler K, Berens C, Geue L, Menge C. Metabolic traits of bovine shiga toxinproducing escherichia coli (STEC) strains with different colonization properties. Toxins (Basel). 2020;12(6).

74. Stecher B. Establishing causality in Salmonella-microbiota-host interaction: The use of gnotobiotic mouse models and synthetic microbial communities. Int J Med Microbiol. 2021;311(3).

75. Ma KC, Mortimer TD, Hicks AL, Wheeler NE, Sánchez-Busó L, Golparian D, et al. Adaptation to the cervical environment is associated with increased antibiotic susceptibility in Neisseria gonorrhoeae. Nat Commun. 2020;

76. Gröschel MI, Meehan CJ, Barilar I, Diricks M, Gonzaga A, Steglich M, et al. The phylogenetic landscape and nosocomial spread of the multidrug-resistant opportunist Stenotrophomonas maltophilia. Nat Commun. 2020;

77. Gao Q, Wang X, Xu H, Xu Y, Ling J, Zhang D, et al. Roles of iron acquisition systems in virulence of extraintestinal pathogenic Escherichia coli: Salmochelin and aerobactin contribute more to virulence than heme in a chicken infection model. BMC Microbiol. 2012;

78. McPhee JB, Small CL, Reid-Yu SA, Brannon JR, Moual H Le, Coombes BK. Host defense peptide resistance contributes to colonization and maximal intestinal pathology by Crohn's disease-associated adherent-invasive Escherichia coli. Infect Immun. 2014;

79. Fjell CD, Jenssen H, Fries P, Aich P, Griebel P, Hilpert K, et al. Identification of novel host defense peptides and the absence of $\alpha$-defensins in the bovine genome. Proteins Struct Funct Genet. 2008;

80. Lynn DJ, Higgs R, Gaines S, Tierney J, James T, Lloyd AT, et al. Bioinformatic discovery and initial characterisation of nine novel antimicrobial peptide genes in the chicken. Immunogenetics. 2004;

81. Li Z, Hu Y, Yang Y, Lu Z, Wang Y. Antimicrobial resistance in livestock: Antimicrobial peptides provide a new solution for a growing challenge. Anim Front. 2018;

82. Liu Q, Yao S, Chen Y, Gao S, Yang Y, Deng J, et al. Use of antimicrobial peptides as a feed additive for juvenile goats. Sci Rep. 2017; 
bioRxiv preprint doi: https://doi.org/10.1101/2022.02.08.479532; this version posted February 9,2022 . The copyright holder for this preprint (which was not certified by peer review) is the author/funder, who has granted bioRxiv a license to display the preprint in perpetuity. It is made available under aCC-BY-NC 4.0 International license.

691
83. Xiao H, Shao F, Wu M, Ren W, Xiong X, Tan B, et al. The application of antimicrobial peptides as growth and health promoters for swine. Journal of Animal Science and Biotechnology. 2015.

84. Barth S, Schwanitz A, Bauerfeind R. Polymerase chain reaction-based method for the typing of f18 fimbriae and distribution of f18 fimbrial subtypes among porcine shiga toxin-encoding escherichia coli in Germany. J Vet Diagnostic Investig. 2011;23(3).

85. Frydendahl K, Jensen TK, Andersen JS, Fredholm M, Evans G. Association between the porcine Escherichia coli F18 receptor genotype and phenotype and susceptibility to colonisation and postweaning diarrhoea caused by E. coli O138:F18. Vet Microbiol. 2003;93(1).

86. Barth S, Tscholshiew A, Menge C, Weiß R, Baljer G, Bauerfeind R. Virulence and fitness gene patterns of Shiga toxin-encoding Escherichia coli isolated from pigs with edema disease or diarrhea in Germany. Berl Munch Tierarztl Wochenschr. 2007;120(7-8).

87. Power RA, Parkhill J, De Oliveira T. Microbial genome-wide association studies: lessons from human GWAS. Nat Rev Genet. 2016;18(1):41-50.

88. Toloza L, Giménez R, Fábrega MJ, Alvarez CS, Aguilera L, Cañas MA, et al. The secreted autotransporter toxin (Sat) does not act as a virulence factor in the probiotic Escherichia coli strain Nissle 1917. BMC Microbiol. 2015; 
bioRxiv preprint doi: https://doi.org/10.1101/2022.02.08.479532; this version posted February 9, 2022. The copyright holder for this preprint (which was not certified by peer review) is the author/funder, who has granted bioRxiv a license to display the preprint in perpetuity. It is made available under aCC-BY-NC 4.0 International license.

738

739

740

741

742

743

744

\section{Figure Legends:}

Fig 1:Distribution of 1198 isolates with host species by a) core-genome phylogeny and b) clustering based on accessory gene content (right). Clades on the phylogeny represent phylogroups, inner-ring represents phylogenetic clusters, middle-ring represents host-species, and outer ring indicates the geographical region.

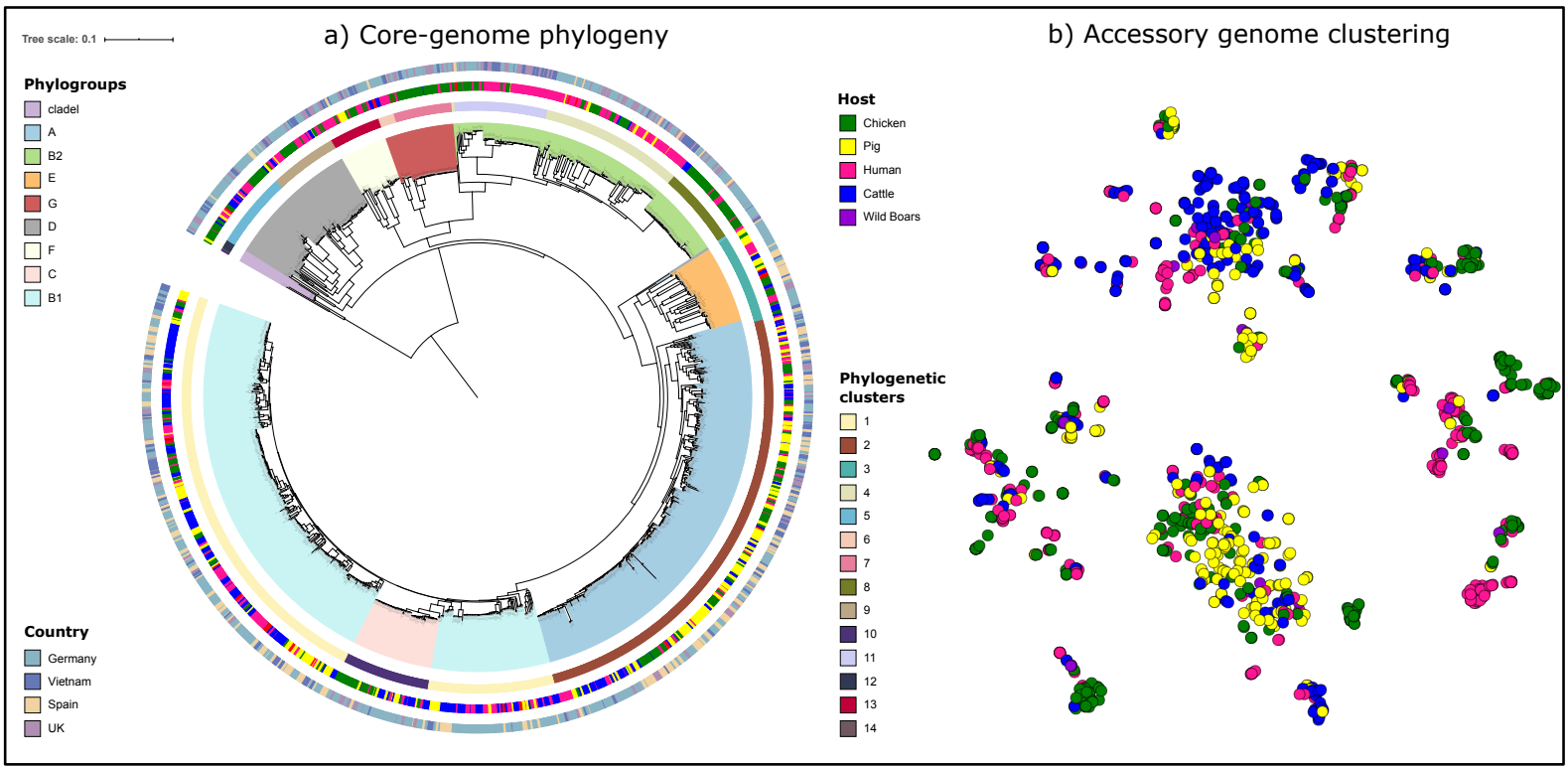

Fig 2: Plots representing the E. coli genes or gene variants associated with the a) Cattle host, b) Human host, and c) Chicken host. The bubble size represents the number of k-mers mapped to a specific gene, and the color gradient represents the effect size $(\beta)$.

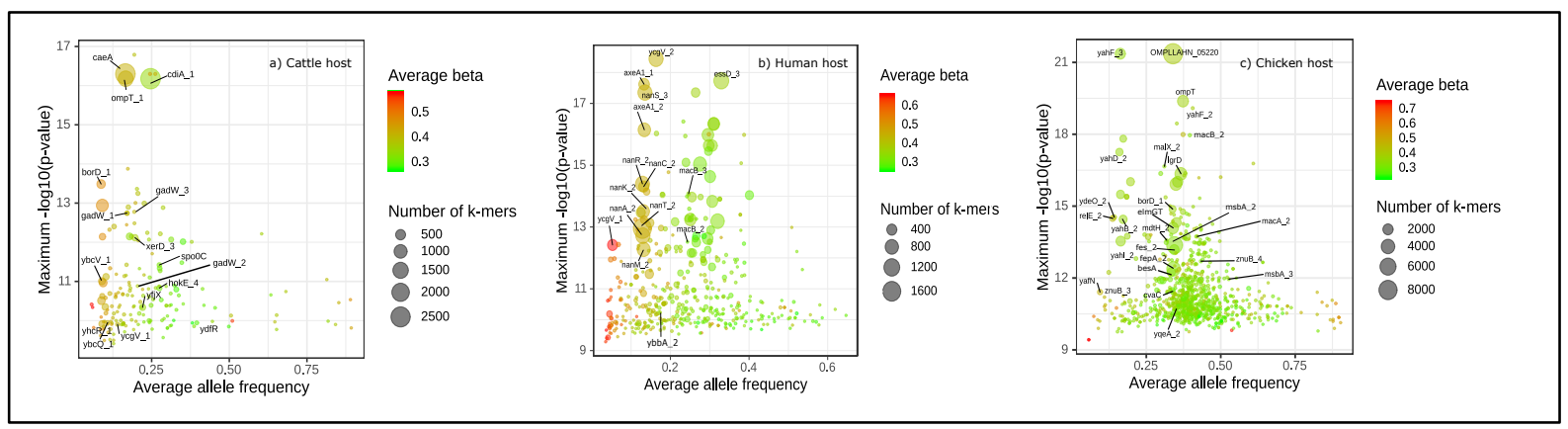

Fig 3: a) Genetic architecture of the human-associated nan gene cluster (nan-9) and the sialoregulon on the complete genome of the strain IMT12185. The strain lacks the nanXY genes of the sialoregulon. b) Distribution of the nan-9 cluster on core-genome phylogeny marked with STs with higher prevalence. c) The table indicates the prevalence of the nan-9 gene cluster in different STs in our collection and in the RefSeq E. coli genomes. 


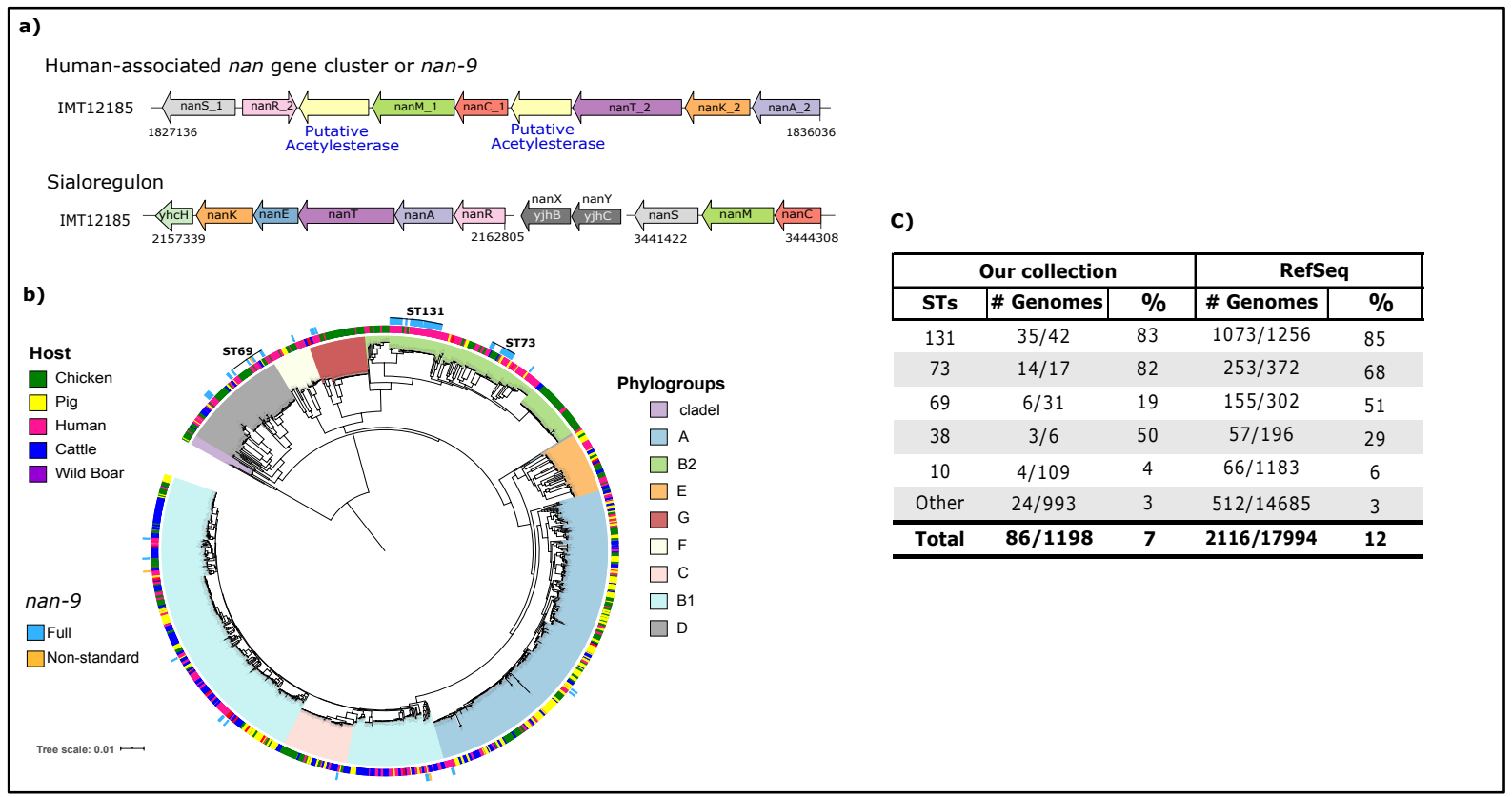

Fig 4: Growth curves of E. coli IMT12185 and its mutant derivatives in various media. a) Growth of IMT12185 and IMT12185 $\Delta$ nan-9 in M9 minimal medium with $0.2 \%$ 5-N-Acetylneuraminic acid (Neu5Ac) b)

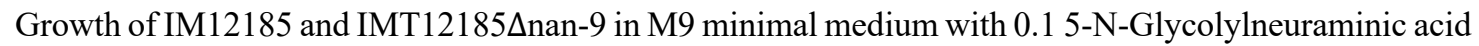
(Neu5Gc) c) Growth of IMT12185 and IMT12185 $\Delta$ nanRATEK in M9 minimal medium with $0.2 \% 5$-NAcetylneuraminic acid (Neu5Ac) d) Growth of IMT12185 and IMT12185 $\Delta$ nan-9 in lysogeny broth (LB).
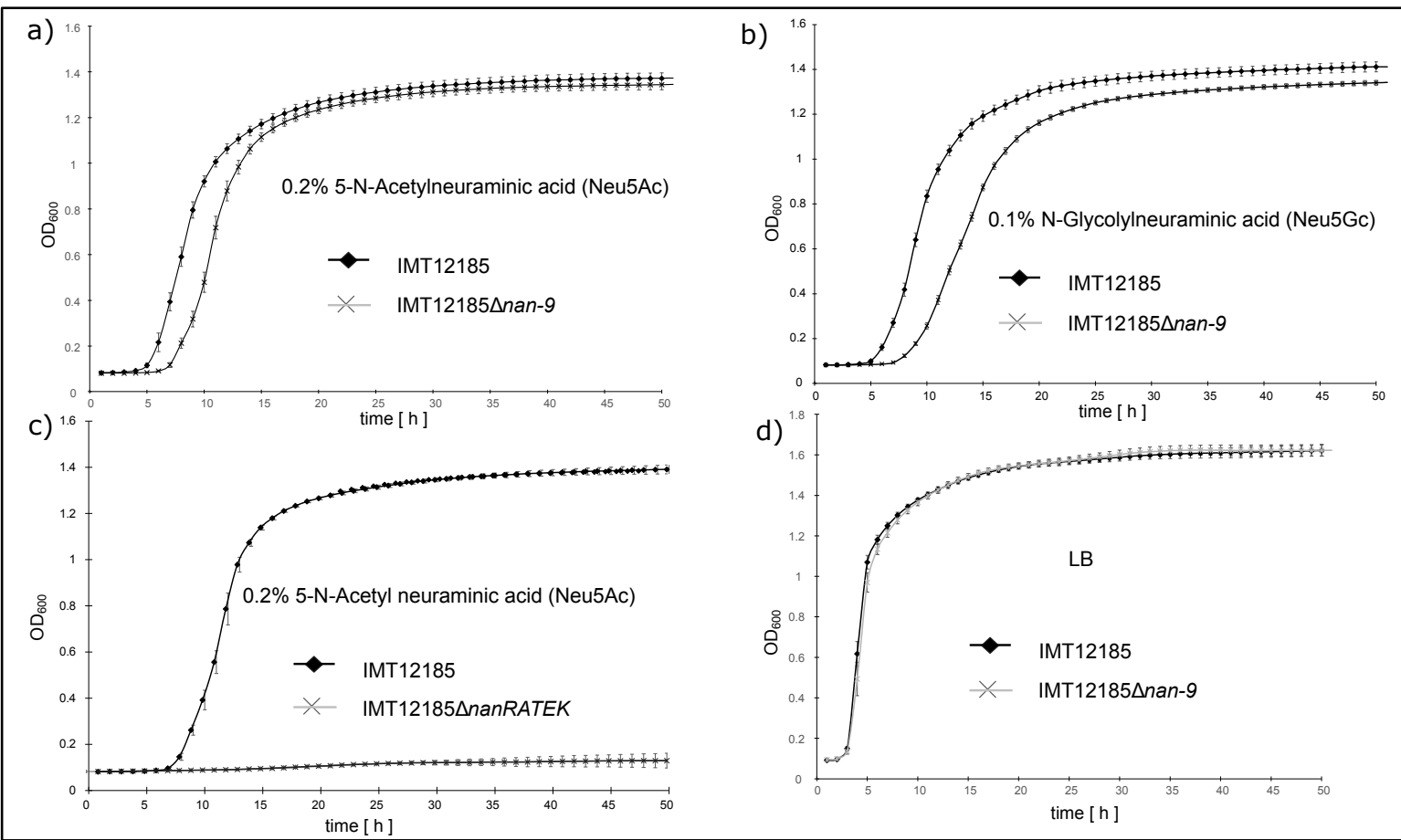
bioRxiv preprint doi: https://doi.org/10.1101/2022.02.08.479532; this version posted February 9, 2022. The copyright holder for this preprint (which was not certified by peer review) is the author/funder, who has granted bioRxiv a license to display the preprint in perpetuity. It is made available under aCC-BY-NC 4.0 International license.

764 Fig 5: Distribution of ompP, $\operatorname{arlC}$, and $о m p T$ genes in phylogroups and host across the phylogeny and their estimated prevalence.

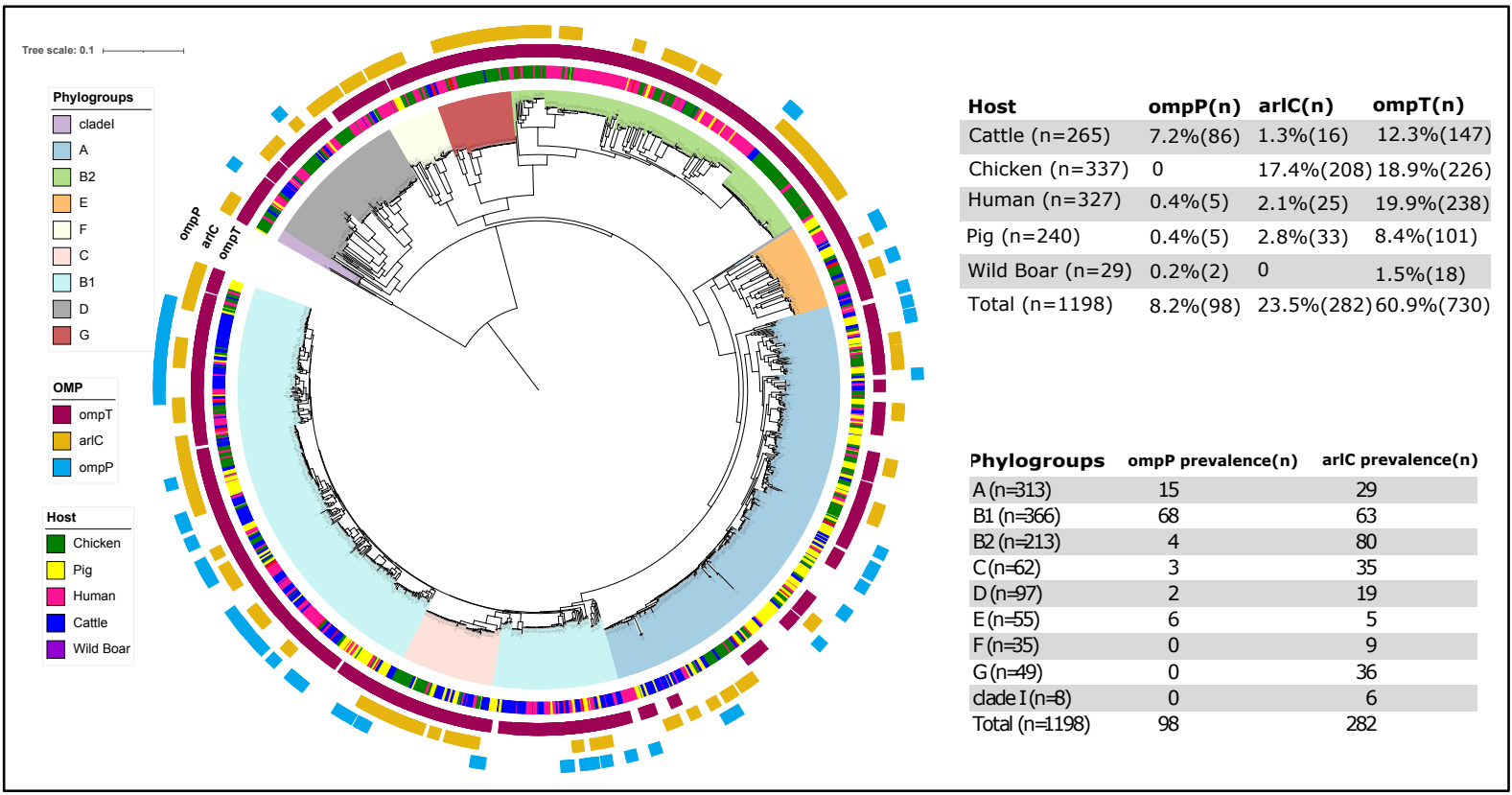

